\title{
Role of adiponectin and free fatty acids on the association between abdominal visceral fat and insulin resistance
}

Aida Medina-Urrutia ${ }^{1}$, Carlos Posadas-Romero', Rosalinda Posadas-Sánchez ${ }^{1}$ Esteban Jorge-Galarza ${ }^{1}$, Teresa Villarreal-Molina ${ }^{3}$, María del Carmen González-Salazar', Guillermo Cardoso-Saldaña', Gilberto Vargas-Alarcón ${ }^{2}$, Margarita Torres-Tamayo ${ }^{1}$ and Juan Gabriel Juárez-Rojas ${ }^{1 *}$

\begin{abstract}
Background: Experimental studies have shown that high free fatty acid (FFA) and low adiponectin (ADIPO) levels are involved in the mechanisms by which adiposity promotes insulin resistance (IR). However, no previous clinical studies have simultaneously analysed the relative contribution of FFA and ADIPO levels on the relation of abdominal visceral fat (AVF) with insulin resistance.
\end{abstract}

Objective: To analyse the contribution of low ADIPO (adiponectin < =p25th: $8.67 \mu \mathrm{g} / \mathrm{mL}$ in women and $5.30 \mu \mathrm{g} / \mathrm{mL}$ in men), and high FFAs (FFAs > =p75th: $0.745 \mathrm{mEq} / \mathrm{L}$ in women and $0.60 \mathrm{mEq} / \mathrm{L}$ in $\mathrm{men}$ ) to the association of high AVF (AVF > =p75th: $127 \mathrm{~cm}^{2}$ in women; $152.7 \mathrm{~cm}^{2}$ in men) with insulin resistance (HOMA-IR > =75th: 3.58 in women and 3.12 in men), in non-diabetic subjects.

Material and methods: A cross-sectional analysis was performed including 1217 control participants of the Genetics of Atherosclerotic Disease study (GEA). Clinical, tomographic and biochemical parameters were measured in all participants. Logistic regression models were used to assess the association of high AVF with IR stratifying according to gender, and to normal or low ADIPO and normal or high FFA serum levels.

Results: In comparison to referent group, in men low ADIPO unlike high FFA increased the risk of IR. Females with normal AVF and low ADIPO, or high AVF and normal ADIPO had aprox 3 folds risk of IR (OR [IC95\%]: 3.7 [2.1-6.6], $\mathrm{p}<0.001$, and 3.4 [2.0-5.7], $\mathrm{p}<0.001$; respectively). The risk increased to 7.6 [4.2-13.8], $\mathrm{p}<0.001$ when high AVF and low ADIPO were present. Irrespective of AVF, the effect of low ADIPO on IR was higher than that seen for high FFA. Besides, our results suggest an additive effect of high AVF, high FFA and low ADIPO on the IR prevalence.

Conclusions: The present study provides novel and important information about the combined effect of high AVF and low ADIPO on the risk of IR. Furthermore, our data suggest that the effect of low adiponectin levels on the high AVF-IR association is stronger than that observed for high FFA, suggesting that adiponectin could be used as biomarker to identify subjects at high risk for T2DM and CAD.

Keywords: Adiponectin, Free fatty acids, Visceral fat, Insulin resistance

\footnotetext{
* Correspondence: gaboyk2@gmail.com

${ }^{1}$ Endocrinology Department, National Institute of Cardiology "Ignacio

Chávez", Juan Badiano No. 1 Col. Sección XVI Tlalpan, C.P, 14080 México City,

México

Full list of author information is available at the end of the article
} 


\section{Introduction}

Coronary artery disease (CAD) and type 2 diabetes mellitus (T2DM) are currently leading causes of mortality worldwide [1]. Although obesity has been recognized as one of the most important risk factors for both these chronic diseases [2], approximately $30 \%$ of overweight or obese subjects [body mass index $(\mathrm{BMI}) \geq 25 \mathrm{Kg} / \mathrm{m}^{2}$ ], do not show metabolic abnormalities [3,4]. Several studies have sought to identify when obesity is associated with metabolic abnormalities and increased risk of developing T2DM and CAD [5,6]. Abdominal visceral fat (AVF) has proven to be a better predictor of metabolic abnormalities (particularly insulin resistance) than BMI and waist circumference [7].

Experimental evidence suggests that high free fatty acid (FFA) [8] and low adiponectin (ADIPO) plasma levels [9] play a key role in the mechanisms by which excess of adiposity promotes insulin resistance $[10,11]$. Nevertheless, few clinical investigations have analysed the effect of high FFA serum levels on the association between increased AVF and insulin resistance (IR) [12]. As far as we know, no previous epidemiological studies have analysed the contribution of ADIPO serum levels on AVF-IR association. Considering that insulin resistance is a crucial feature of the metabolic abnormalities observed in obese subjects, the purpose of the present study was to analyse the role of ADIPO and FFA levels on the association between high AVF and IR in a Mexican-Mestizo population.

Our data suggest that the effect of low adiponectin levels on the high AVF-IR association is stronger than that observed for high FFA, suggesting that adiponectin could be used as biomarker to identify subjects at high risk for T2DM and CAD.

\section{Material and methods Study subjects}

The study population was recruited from controls participating in the Genetics of Atherosclerotic Disease (GEA) study. The GEA study was designed to examine the genomic bases of CAD and to assess traditional and emerging risk factors for clinical and subclinical atherosclerotic vascular disease in the adult Mexican population [13]. All GEA participants are of self-reported Mexican-Mestizo ancestry (Spaniards and Native-American Indians). Briefly, a convenience sample of 1200 CAD patients and 1500 control subjects aged 30 to 75 years was recruited from residents in Mexico City. Patients with established premature CAD were selected from the outpatient clinic of the National Institute of Cardiology. Volunteer control participants with a negative family history of premature CAD and no personal history of cardiovascular disease were recruited from apparently healthy blood donors and through brochures posted in social service centers. Coronary patients and control subjects with history of renal, liver, thyroid or malignant disease, and corticosteroid use were not included. The GEA study was approved by the Institutional Review Board of the National Institute of Cardiology and conducted according to the Declaration of Helsinki. Written informed consent was obtained from participants.

For the purpose of the present study, we included only control subjects without T2DM. All participants answered several structured questionnaires that provide detailed information regarding family history, demographics, diet, physical activity, medications, smoking, and alcohol intake. Systolic and diastolic blood pressures were measured after subjects rest for at least 10 minutes, and the average of the second and third measurements was used as the subject's blood pressure. Height, weight and waist circumference were measured and BMI calculated as weight in kilograms divided by height in meters squared.

\section{Biochemical analyses}

Venous blood samples were collected from subjects after 10 hour fasting. Plasma glucose, total cholesterol, triglycerides and high density lipoprotein cholesterol (HDL-C) were measured in fresh samples, using standardized enzymatic procedures in a Hitachi 902 analyzer (Hitachi LTD, Tokyo, Japan). Accuracy and precision of lipid measurements in our laboratory are under periodic surveillance by the Centers for Disease Control and Prevention Service (Atlanta, GA). Low density lipoprotein cholesterol (LDL-C) was estimated by using the De Long et al. formula [14]. Total high sensitive C-reactive protein (hs-CRP) levels were determined by immunonephelometry on a BN ProSpec nephelometer (Dade Behring, Marburg Hesse, Germany). Inter-assay coefficient of variation was less than $6 \%$ for nephelometric determinations. Serum FFA were measured by an enzymaticcolorimetric assay (Wako Diagnostics, Chuo-Ku Osaka Japan) in a Hitachi 902 auto analyser, with intra and inter-assay variation coefficients below $3 \%$. Total serum adiponectin was measured by Enzyme Linked Immunosorbent Assay (ELISA) technique ( $R$ \& D Systems Quantikne Kit, Minneapolis, Minnesota, USA) with intra and inter-assay variation coefficients lower than $10 \%$. Plasma insulin concentrations were determined by a radioimmunoassay (Millipore; RIA Kit, Cat. No. HI-14 K, St. Charles, Missouri, USA), the intra and inter-assay variation coefficients were $2.1 \%$ and $6.8 \%$, respectively. Insulin resistance was estimated using the homeostasis model assessment $($ HOMA-IR $=$ insulin $[\mu \mathrm{IU} / \mathrm{mL}] \mathrm{X}$ glucose $\mathrm{mmol} / 22.5)$ [15]. Percentiles for insulin resistance, FFA and adiponectin levels were established in a subsample of the GEA control group (127 men and 169 
women) without T2DM (defined as fasting plasma glucose $\geq 126 \mathrm{mg} / \mathrm{dL}$, prior medical diagnosis, or antidiabetic treatment use) [16], and without cardiometabolic risk factors, namely $\mathrm{BMI}<30 \mathrm{~kg} / \mathrm{m}^{2}$, blood pressure $<$ $140 / 90 \mathrm{mmHg}$, fasting glucose $<100 \mathrm{mg} / \mathrm{dL}, \mathrm{HDL}-\mathrm{C}>$ $40 \mathrm{mg} / \mathrm{dL}$ in men and $>50 \mathrm{mg} / \mathrm{dL}$ in women, and triglyceride levels $<150 \mathrm{mg} / \mathrm{dL}$. Insulin resistance and high FFA levels were defined as HOMA-IR and FFA levels $\geq 75$ th percentile (HOMA-IR: $>3.58$ in women and $>3.12$ in men; FFA levels: $>0.745 \mathrm{mEq} / \mathrm{L}$ in women and $>0.60 \mathrm{mEq} / \mathrm{L}$ in men), while low ADIPO was defined as serum adiponectin levels $\leq 25$ th percentile $(\leq 8.67 \mu \mathrm{g} / \mathrm{mL}$ in women and $\leq 5.30 \mu \mathrm{g} / \mathrm{mL}$ in men).

\section{Computerized axial tomography study}

Abdominal subcutaneous and visceral fat were quantified by computed tomography in a tomographic abdomen slice at the L4-L5 inter vertebral space. Computed tomography was performed using a 64-channel multi-detector helical computed tomography system (Somatom Sensation, Siemens. Forciem, Bavaria, Germany) and interpreted by experienced radiologists as described by Kvist et al. [17]. Elevated AVF was defined as AVF $\geq 75$ th percentile (127 $\mathrm{cm}^{2}$ in women; $152.7 \mathrm{~cm}^{2}$ in men). This cut-off point was also estimated in the sub-group of GEA participants without cardiometabolic risk factors.

\section{Statistical analysis}

Data are presented as mean $\pm \mathrm{SD}$, median (interquartile range), or prevalence for categorical variables. Comparisons were made by $t$ test, ANOVA and Sheffe as Post hoc, U Mann-Whitney, Kruskal-Wallis or Chi-squared test, as appropriate. In order to explore whether low ADIPO levels affect the AVF-IR association, the study population was stratified in four groups as follows: 1) Reference group: AVF $<$ p75 (normal AVF) and adiponectin $>$ p25 (normal ADIPO), 2) subjects with normal AVF and low ADIPO, 3)subjects with high AVF and normal ADIPO, and 4) subjects with high AVF and low ADIPO levels. To study the effect of the FFA levels on this association, the following groups were compared: 1 ) Reference group: normal AVF and FFA < p75 (normal FFA), 2) subjects with normal AVF and high FFA, 3) subjects with high AVF and normal FFA, and 4) subjects with high AVF and high FFA. Logistic regression models were used to analyse the effect of low ADIPO and high FFA levels on the association of high AVF with insulin resistance. Age, BMI, hs-CRP, triglycerides, HDL-C, glucose, total caloric intake, and total physical activity, were included as co-variables. The interaction of ADIPO and FFA levels with AVF was evaluated by including the AVF*FFAs or AVF*ADIPO interaction terms in the logistic regression models [18]. All $\mathrm{p}$ values $<0.05$ were considered as statistically significant. The analyses were carried out with the statistical software SPSS v15.0 (SPSS Chicago, II).

\section{Results}

The present study included 1217 GEA control subjects (50.5\% women), with a mean age of $52.5 \pm 9.0$ years, BMI of $28.3 \pm 4.3 \mathrm{Kg} / \mathrm{m}^{2}$, and HOMA-IR median of 3.7 (2.6-5.3). Women had higher subcutaneous abdominal fat volume [320 (256-396) $\mathrm{cm}^{2}$ vs. $243(183-307) \mathrm{cm}^{2}$; $\mathrm{p}<0.0001$ ] and lower AVF volume than men [124 (93166) $\mathrm{cm}^{2}$ vs. $\left.168(129-217) \mathrm{cm}^{2} ; \mathrm{p}<0.0001\right]$. Women also had higher FFA $[0.61(0.49-0.76) \mathrm{mEq} / \mathrm{L}$ vs. 0.49 $(0.39-0.61) \mathrm{mEq} / \mathrm{L} ; \mathrm{p}<0.0001]$ and higher adiponectin levels as compared to men [10.4 (6.7-15.7) $\mu \mathrm{g} / \mathrm{mL}$ vs. 6.2 (4.0-9.4) $\mu \mathrm{g} / \mathrm{mL} ; \mathrm{p}<0.0001]$.

Table 1 shows the clinical characteristics of the study population stratified according to gender, normal and high AVF, and normal and low ADIPO levels. The prevalence of low ADIPO levels in individuals with normal AVF was $28.2 \%$ in women and $32.9 \%$ in men, and this prevalence was significantly higher in individuals with high AVF (40.9\% in women and $44.7 \%$ in men). Normal AVF/low ADIPO individuals had significantly higher BMI and triglyceride levelsas well as lower HDL-C levels (in both genders), and significantly higher diastolic blood pressure and glucose levels (only in men) as compared to the reference group $(\mathrm{p}<0.05)$. High AVF was associated with a less favorable metabolic profile in both genders, and high AVF/low ADIPO individuals showed the lowest HDL-C and the highest triglyceride concentrations among all study groups. Similarly, on stratifying according to AVF and FFA levels, high AVF/high FFA individuals showed the most unfavorable metabolic profile (data not shown), although only increased systolic blood pressure was statistically significant on comparison with the high AVF/normal FFA group, [women: 116 (108-130) $\mathrm{mmHg}$ vs. 111 (103-123) mmHg, p < 0.05; men: 123 (115-131) $\mathrm{mmHg}$ vs. 118 (111-129) $\mathrm{mmHg}$, $\mathrm{p}<0.05]$.

In women, high AVF, low ADIPO and high FFA were all significantly associated with a higher prevalence of IR (Figure 1). Moreover, high AVF/low ADIPO and high AVF/high FFA combinations showed a stronger association with IR than any of these parameters assessed individually. In contrast, the prevalence of IR in men with high AVF was not significantly affected by low ADIPO or high FFA levels as assessed by univariate analysis (Figure 1), although logistic regression analyses adjusting for confounding factors, revealed that low ADIPO, but not high FFA, significantly increases the risk of IR in men with high AVF (Figure 1 and Table 2). Moreover, normal AVF/low ADIPO and high AVF/normal ADIPO women had a 3 fold increased risk of IR even after controlling for FFA levels and other confounding 
Table 1 Clinical characteristics of study subjects stratified by sex, abdominal visceral fat, and adiponectin

\begin{tabular}{|c|c|c|c|c|}
\hline & \multicolumn{2}{|l|}{ Normal AVF } & \multicolumn{2}{|l|}{ High AVF } \\
\hline & Normal ADIPO & Low ADIPO & Normal ADIPO & Low ADIPO \\
\hline \multicolumn{5}{|l|}{ WOMEN } \\
\hline N & 211 & 83 & 189 & 131 \\
\hline Age (years) & $51 \pm 9$ & $49 \pm 9$ & $56 \pm 8^{*^{\dagger}}$ & $52 \pm 8^{\dagger \neq}$ \\
\hline BMI $\left(\mathrm{kg} / \mathrm{m}^{2}\right)$ & $25(23-28)$ & $26(24-29)^{*}$ & $30(27-33)^{* \dagger}$ & $31(28-34)^{* \dagger}$ \\
\hline $\mathrm{SBP}(\mathrm{mmHg})$ & 106(97-118) & 103(99-111) & $113(105-124)^{*^{+}}$ & $111(105-123)^{*^{\dagger}}$ \\
\hline $\mathrm{DBP}(\mathrm{mmHg})$ & $67(62-73)$ & $66(60-71)$ & $71(66-76)^{*^{\dagger}}$ & $70(65-76)^{*^{\dagger}}$ \\
\hline LDL-C (mmol/L) & $2.98 \pm 0.75$ & $3.13 \pm 0.98$ & $3.16 \pm 0.83$ & $2.98 \pm 0.83$ \\
\hline $\mathrm{HDL}-\mathrm{C}(\mathrm{mmol} / \mathrm{L})$ & $1.47 \pm 0.36$ & $1.27 \pm 0.34^{*}$ & $1.32 \pm 0.36^{*}$ & $1.14 \pm 0.31^{* \neq}$ \\
\hline TG $(\mathrm{mmol} / \mathrm{L})$ & 1.25(0.95-1.6) & $1.59(1.2-2.1)^{*}$ & $1.65(1.3-2.2)^{*}$ & $1.76(1.4-2.3)^{*}$ \\
\hline Glucose (mmol/L) & 4.66(4.4-4.9) & $4.77(4.5-5.1)$ & $5.05(4.7-5.3)^{* \dagger}$ & $5.05(4.7-5.4)^{*^{\dagger}}$ \\
\hline HOMA-IR & $2.5(2.0-3.4)$ & $3.6(2.4-4.7)^{*}$ & $4.3(3.2-5.9)^{*^{\dagger}}$ & $5.0(3.9-6.9) *{ }^{+\ddagger}$ \\
\hline hs-CRP (nmol/L) & $10.5(5.7-21.9)$ & $12.4(6.7-31.4)$ & $23.8(12.4-39.0)^{*^{\dagger}}$ & $28.6(12.4-45.7)^{*^{+}}$ \\
\hline \multicolumn{5}{|l|}{ MEN } \\
\hline N & 157 & 77 & 204 & 165 \\
\hline Age (years) & $51 \pm 11$ & $48 \pm 9$ & $55 \pm 10^{*+}$ & $52 \pm 8^{+}$ \\
\hline BMI $\left(\mathrm{kg} / \mathrm{m}^{2}\right)$ & $25(23-27)$ & $26(25-29)^{*}$ & $29(27-32)^{*^{\dagger}}$ & $29(27-32)^{*^{\dagger}}$ \\
\hline $\mathrm{SBP}(\mathrm{mmHg})$ & 112(106-119) & 115(107-124) & $121(111-133)^{* \dagger}$ & $119(112-127)^{*^{\dagger}}$ \\
\hline $\mathrm{DBP}(\mathrm{mmHg})$ & $69(65-75)$ & $72(68-76)^{*}$ & $75(70-82)^{* \dagger}$ & $75(70-81)^{* \dagger}$ \\
\hline LDL-C (mmol/L) & $2.98 \pm 0.70$ & $3.03 \pm 0.75$ & $3.19 \pm 0.85$ & $3.16 \pm 0.93$ \\
\hline $\mathrm{HDL}-\mathrm{C}(\mathrm{mmol} / \mathrm{L})$ & $1.19 \pm 0.28$ & $1.04 \pm 0.28^{*}$ & $1.06 \pm 0.26^{*}$ & $0.98 \pm 0.23^{* \ddagger}$ \\
\hline TG (mmol/L) & $1.34(0.97-2.0)$ & $1.68(1.2-2.5)^{*}$ & $1.90(1.5-2.6)^{*^{+}}$ & $2.07(1.56-3.01)^{*+\neq}$ \\
\hline Glucose (mmol/L) & $4.83(4.7-5.2)$ & $5.05(4.7-5.3)^{*}$ & $5.05(4.7-5.4)^{*}$ & $5.16(4.9-5.4)^{* \dagger}$ \\
\hline HOMA-IR & $2.5(1.9-3.3)$ & $3.2(2.2-4.8)^{*}$ & $4.3(3.0-5.7)^{* \dagger}$ & $5.1(3.4-6.7) *+\neq$ \\
\hline hs-CRP (nmol/L) & $8.6(4.8-16.2)$ & $9.5(5.7-18.1)$ & $13.3(7.6-25.7)^{*}$ & $15.2(8.6-28.6)^{*^{\dagger}}$ \\
\hline
\end{tabular}

Mean \pm SD or median (interquartil range); ANOVA or Kruskal-Wallis. ${ }^{*} p<0.05$ vs. normal AVF/ normal ADIPO; ${ }^{\dagger} p<0.05$ vs. normal AVF/low ADIPO; ${ }^{\ddagger} p<0.05$ vs. high AVF/normal ADIPO. AVF = Abdominal visceral fat; $\mathrm{ADIPO}=$ Adiponectin; $\mathrm{BMI}=$ Body mass index; $\mathrm{SBP}=\mathrm{Systolic}$ blood pressure; $\mathrm{DBP}=\mathrm{Diastolic}$ blood pressure; $\mathrm{LDL}-\mathrm{C}=\mathrm{Low}$ density lipoprotein cholesterol cholesterol; $\mathrm{HDL}-\mathrm{C}=$ High density lipoprotein cholesterol; $\mathrm{TG}=$ Triglycerides; $\mathrm{hs}$ - $\mathrm{CRP}=\mathrm{High}$ sensitivity $\mathrm{C}$-reactive protein.

variables. The odds ratio for IR increased to 7.6 in the high AVF/low ADIPO group. Irrespective of the AVF, the effect of low ADIPO on IR was higher than that observed for high FFA levels. Because interactions terms AVF*ADIPO or AVF*FFA were not statistically significant in the logistic regression models, the results suggest an additive and independent effect of AVF, ADIPO and FFA levels on the prevalence of IR.

\section{Discussion}

This study shows for the first time that irrespective of AVF, low ADIPO and high FFA levels are associated with IR, and that the effect of low ADIPO is stronger than that of high FFA levels. Moreover, an additive effect of low ADIPO and high FFAs with high AVF on IR prevalence was observed. While subjects with high AVF or low ADIPO had a three-fold increased risk of IR, the combination of low ADIPO with high AVF doubled this probability. Interestingly, high FFA levels were associated with IR only in females, but were significantly associated with IR in both genders when combined with high AVF. Our results suggest that high AVF, high FFA and low ADIPO levels have an additive and independent effect increasing the risk of IR. This is particularly relevant because insulin resistance is a common alteration among obese subjects, and that both abnormalities are precursors of T2DM and CAD.

Adiponectin is a constitutively secreted adipocyte protein. In obese individuals, ADIPO serum levels are frequently low and show an inverse correlation with AVF, but not with BMI or subcutaneous fat deposits $[19,20]$. It has been suggested that this protein is a potent insulin-sensitizing agent [9,21]. Aguilar-Salinas et al. [22] reported that obese subjects with high ADIPO levels had lower insulin concentrations and a better metabolic profile, as compared to obese subjects with low ADIPO levels. Our results extend those observations, by suggesting that low ADIPO levels are associated with a higher risk of IR, 


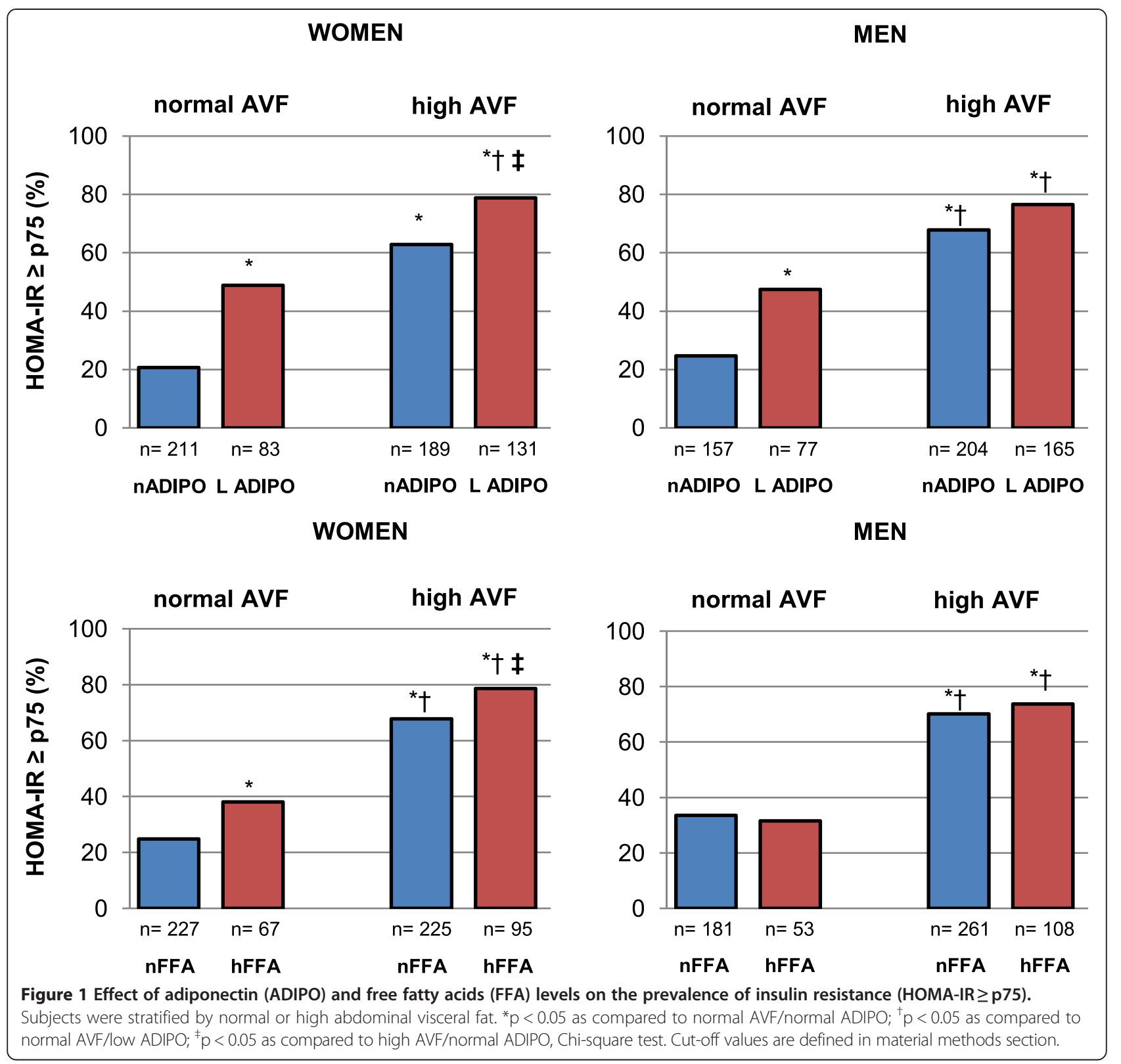

Table 2 Regression logistic models* analyzing the effect of AVF, adiponectin and FFA on insulin resistance prevalence

\begin{tabular}{|c|c|c|c|c|c|}
\hline & \multicolumn{2}{|l|}{ Normal AVF } & \multicolumn{2}{|l|}{ High AVF } & \multirow[b]{2}{*}{ Interaction $\mathrm{p}$} \\
\hline & Normal ADIPO & Low ADIPO & Normal ADIPO & Low ADIPO & \\
\hline Women & 1 & $3.7(2.1-6.6)$ & $3.4(2.0-5.7)$ & $7.6(4.2-13.8)$ & 0.6 \\
\hline \multirow[t]{3}{*}{ Men } & 1 & $1.9(1.0-3.6)$ & $2.5(1.4-4.3)$ & $4.1(2.6-7.2)$ & 0.7 \\
\hline & \multicolumn{2}{|l|}{ Normal AVF } & \multicolumn{2}{|l|}{ High AVF } & \\
\hline & Normal FFA & High FFA & Normal FFA & High FFA & \\
\hline Women & 1 & $2.2(1.1-4.1)$ & $2.8(1.7-4.6)$ & $4.5(2.3-8.8)$ & 0.5 \\
\hline Men & 1 & $0.8(0.4-1.8)$ & $2.1(1.3-3.4)$ & $2.3(1.2-4.2)$ & 0.6 \\
\hline
\end{tabular}

${ }^{*}$ Age, body mass index, high sensitive C-reactive protein, physical activity, total caloric intake, and FFA or adiponectin levels were included as co-variables (similar results were obtained after including triglycerides, $\mathrm{HDL}-\mathrm{C}$ and glucose plasma levels in the model). AVF $=\mathrm{Abdominal}$ visceral fat; $\mathrm{FFA}=\mathrm{Free}$ fatty acids; $\mathrm{ADIPO}=$ Adiponectin; HDL-C = High density lipoprotein cholesterol. Cut-off values are defined in material method section. 
independently of AVF and other confounding factors. Moreover, we found that the high AVF/low ADIPO combination has a synergic effect on IR risk. These findings are supported by in vitro and in vivo studies showing that the interaction of adiponectin with its cellular receptors (AdipoR1 and AdipoR2) activates AMP kinase promoting the translocation of GLUT4 transporters, simultaneously reduces hepatic glucose production by inhibiting the enzyme phosphoenolpyruvate carboxylase, inhibits the synthesis of fatty acids and stimulates their oxidation [21]. Adiponectin also acts as an agonist of peroxisome proliferator activated receptor (PPAR) gamma, leading additional uptake of plasma glucose [21], and enhances insulin sensitivity by increasing hepatic insulin receptor substrate 2 (IRS-2). It has been also proposed that highmolecular adiponectin binds to membrane T-cadherin of adipocytes, forming intercellular spacers and increasing the metabolic activity and insulin sensitivity of these cells [23]. Thus, all these functions confer to adponectin a key role as an insulin sensitizer in muscle, liver, and adipose tissue $[21,23]$.

Several studies have shown that FFA levels are frequently high in obese individuals [10,11]. Excessive FFA release by AVF is particularly deleterious because it exposes the liver to increased FFA levels via portal circulation, impairing insulin liver metabolism and promoting IR [24]. However, studies analyzing the role of FFA on the association of AVF with IR are scarce. While Milleret al. [12] reported that FFAs do not participate in this association, our results show that women with high AVF/ high FFA levels have a twofold increased risk of IR than that of women with high AVF/normal FFA levels (Table 2). The lack of association in men could be explained by the lower FFA levels observed in this gender, and because several lines of evidence show that women are more susceptible to the unfavorable metabolic effects of obesity [25]. Of note, Miller et al. included both men and women in his analyses, and did not analyse genders separately. Thus, gender differences may explain the discrepancies between both studies. Our results are supported by experimental studies showing a liver lipotoxic effect of high FFA [11]. High FFA impair the insulin signalling pathway by decreasing GLUT4 transporter expression [26,27], and favouring ceramide and diacylglycerol production [28].

No previous study has simultaneously analyzed the effect of low ADIPO and high FFA levels on the high AVF-IR association. The results of the present study suggest that both low ADIPO and high FFA play a role in this through independent pathways and related mechanisms. These results are supported by a previous study showing an association of adiponectin with IR, regardless of the circulating FFA [29]. Moreover, our data extend this information by showing that the effect of low
ADIPO on IR is independent of AVF, and that its effect on IR is stronger than that observed for high FFA. Although these associations need to be confirmed in further studies, it is important to point out that low adiponectin concentrations, alone or combined with high AVF, could be considered a major biomarker of IR.

\section{Strengths and limitations}

This study has several strengths. Firstly, the distribution of body fat was measured by computed tomography, which is a specific and reliable method to quantify AVF. It was thus possible to assess the role of high AVF on low ADIPO, high FFA and IR, above and beyond clinical anthropometry. Secondly, the sample size of the study population was large enough and both sexes were included; which is important because female gender has been shown to be more susceptible to unfavorable metabolic effects of obesity. Third, multivariable analyses included physical activity and caloric intake, known to affect ADIPO, FFA levels, AVF and IR. Among the study limitations, it should be mentioned that because this is across-sectional design we cannot infer causality from the results. Moreover, only total adiponectin was measured, and not its multimeric forms which are critical determinants of IR. Nevertheless, because circulating levels of high molecular adiponectin have been reported to show a strong correlation with total adiponectin circulating levels $(r=0.95, p<0.0001)$ [30], the associations observed here would be expected to be similar. Insulin resistance was estimated by the HOMA-IR. This algorithm is as surrogate marker of IR, more feasible for large population studies, which correlate with the euglycemic CLAMP, the gold standard for assessing IR [31]. Finally, it should be noted that since there are no populationspecific thresholds for HOMA-IR, adiponectin, and AVF, we used specific cut-off values obtained in our MexicanMestizo population, as Bonora et al. [32] and The European Group for the study of Insulin Resistance have recommended [33]. Because differences in metabolic markers measurements are well documented among different ethnic groups [34,35], these cut-off values cannot be applied to other population. Using population specific cutoff values may contribute to partly explain discrepancies in associations among different studies and populations.

\section{Conclusions}

The results of the present study provide novel and important information about the combined effect of high AVF and low ADIPO levels on the prevalence of IR. Furthermore, our data suggest that the effect of adiponectin on the high AVF-IR association is stronger than the effect of high FFA levels. Therefore, adiponectin could be used as biomarker to identify subjects at high risk forT2DM and CAD. 


\section{Abbreviations}

CAD: Coronary artery disease; T2DM: Type 2 diabetes mellitus; BMI: Body mass index; AVF: Abdominal visceral fat; IR: Insulin resistance; GEA: Genetics of Atherosclerotic Disease; HDL-C: High density lipoprotein cholesterol; LDL-C: Low density lipoprotein cholesterol; hs-CRP: High sensitive C-reactive protein; FFAs: Free fatty acids; HOMA-IR: Homeostasis model assessment for insulin resistance; ADIPO: Adiponectin.

\section{Competing interest}

The authors declare that they have no competing interests.

\section{Authors' contributions}

AMU, CPR and JGJR- designed the study, were responsible of the data analysis and extraction, and wrote the manuscript with final comment and approval by all the authors. TVM- has been involved in the critical revision of the manuscript for important intellectual content. RPS, EJG, MCGS, GCS, GVA, and MTT- provide data and advice regarding study design. All authors were involved in the drafting of the manuscript. All authors read and approved the final manuscript.

\section{Acknowledgements}

The authors would like to thank to the participants included in the GEA study, and to the staff who has contributed to the proper development of this study.

This project was supported by the National Council for Science and Technology (CONACYT GRANT: SALUD-2010-2-150537).

\section{Author details}

${ }^{1}$ Endocrinology Department, National Institute of Cardiology "Ignacio Chávez", Juan Badiano No. 1 Col. Sección XVI Tlalpan, C.P, 14080 México City, México. ${ }^{2}$ Molecular Biology Department, National Institute of Cardiology "Ignacio Chávez", Mexico City, Mexico. ${ }^{3}$ Laboratorio de Genómica de Enfermedades Cardiovasculares, Instituto Nacional de Medicina Genómica (INMEGEN), Mexico City, Mexico.

\section{Received: 19 November 2014 Accepted: 25 January 2015 \\ Published online: 10 February 2015}

\section{References}

1. World and Health Organization (2014). The 10 leading causes of death in the world, 2000 and 2012 [WWW document]. URL www.who.int/ mediacentre/factsheets/fs310/en/

2. Meigs JB, Wilson PW, Fox CS, Vasan RS, Nathan DM, Sullivan LM, et al. Body mass index, metabolic syndrome, and risk of type 2 diabetes or cardiovascular disease. J Clin Endocrinol Metab. 2006:91:2906-12.

3. Ortega FB, Lee DC, Katzmarzyk PT, Ruiz JR, Sui X, Church TS, et al. The intriguing metabolically healthy but obese phenotype: cardiovascular prognosis and role of fitness. Eur Heart J. 2013;34:389-97.

4. Stefan N, Häring HU, Hu FB, Schulze MB. Metabolically healthy obesity: epidemiology, mechanisms, and clinical implications. Lancet Diabetes Endocrinol. 2013;1:152-62.

5. Fox CS, Massaro JM, Hoffmann U, Pou KM, Maurovich-Horvat P, Liu CY, et al. Abdominal visceral and subcutaneous adipose tissue compartments: association with metabolic risk factors in the Framingham Heart Study. Circulation. 2007;116:39-48.

6. Carr DB, Utzschneider KM, Hull RL, Kodama K, Retzlaff BM, Brunzell JD, et al. Intra-abdominal fat is a major determinant of the National Cholesterol Education Program Adult Treatment Panel III criteria for the metabolic syndrome. Diabetes. 2004;53:2087-94.

7. Messier V, Karelis AD, Prud'homme D, Primeau V, Brochu M, Rabasa-Lhoret R. Identifying metabolically healthy but obese individuals in sedentary postmenopausal women. Obesity. 2010;18:911-7.

8. Perseghin G, Petersen K, Shulman Gl. Cellular mechanism of insulin resistance: potential links with inflammation. Int J Obes Relat Metab Disord. 2003;27:S6-11.

9. Arita Y, Kihara S, Ouchi N, Takahashi M, Maeda K, Miyagawa J, et al. Paradoxical decrease of an adipose-specific protein, adiponectin, in obesity. Biochem Biophys Res Commun. 2012;425:560-4.

10. Johnson AM, Olefsky JM. The origins and drivers of insulin resistance. Cell. 2013;152:673-84

11. Capurso C, Capurso A. From excess adiposity to insulin resistance: the role of free fatty acids. Vascul Pharmacol. 2012;57:91-7.
12. Miller MR, Pereira RI, Langefeld CD, Lorenzo C, Rotter Jl, Chen YD, et al. Levels of free fatty acids (FFA) are associated with insulin resistance but do not explain the relationship between adiposity and insulin resistance in Hispanic Americans: the IRAS Family Study. J Clin Endocrinol Metab. 2012;97:3285-91

13. Villarreal-Molina T, Posadas-Romero C, Romero-Hidalgo S, Antúnez-Argüelles E, Bautista-Grande A, Vargas-Alarcón G, et al. The ABCA1 gene R230C variant is associated with decreased risk of premature coronary artery disease: the genetics of atherosclerotic disease (GEA) study. PLoS One. 2012;7:e49285.

14. DeLong DM, DeLong ER, Wood PD, Lippel K, Rifkind BM. A comparison of methods for the estimation of plasma low- and very low-density lipoprotein cholesterol.The Lipid Research Clinics Prevalence Study. JAMA. 1986:256:2372-7.

15. Matthews DR, Hosker JP, Rudenski AS, Naylor BA, Treacher DF, Turner RC Homeostasis model assessment insulin resistance and beta-cell function from fasting plasma glucose and insulin concentration in man. Diabetologia. 1985:28:412-9.

16. American Diabetes Association. Standards of Medical Care in Diabetes 2010. Diabetes Care. 2010;33:S11-61.

17. Kvist H, Chowdhury B, Grangard U, Tylén U, Sjöström L. Total and visceral adipose-tissue volumes derived from measurement with computed tomography in adult men and women: predicted equations. Am J Clin Nutr. 1988:48:1351-61.

18. Oka R, Kobayashi J, Inazu A, Yagi K, Miyamoto S, Sakurai M, et al. Contribution of visceral adiposity and insulin resistance to metabolic risk factors in Japanese men. Metabolism. 2010;59:748-54.

19. Kishida K, Kim KK, Funahashi T, Matsuzawa Y, Kang HC, Shimomura I. Relationships between circulating adiponectin levels and fat distribution in obese subjects. J AtherosclerThromb. 2011;18:592-5.

20. Matsushita Y, Nakagawa T, Yamamoto S, Kato T, Ouchi T, Kikuchi N, et al. Adiponectin and visceral fat associate with cardiovascular risk factors. Obesity. 2014;22:287-91.

21. Fisman EZ, Tenenbaum A. Adiponectin: a manifold therapeutic target formetabolic syndrome, diabetes, and coronary disease? Cardiovasc Diabetol. 2014;13:103.

22. Aguilar-Salinas CA, García EG, Robles L, Riaño D, Ruiz-Gomez DG, GarcíaUlloa AC, et al. High adiponectin concentrations are associated with the metabolically healthy obese phenotype. J Clin Endocrinol Metab. 2008;93:4075-9.

23. Nakamura MT. YudellBE Loor JJ. Regulation of energy metabolism by long-chain fatty acids. Prog Lipid Res. 2014;53:124-44.

24. Björntorp P. "Portal" adipose tissue as a generator of risk factors for cardiovascular disease and diabetes. Arteriosclerosis. 1990;10:493-6.

25. He H, Ni Y, Chen J, Zhao Z, Zhong J, Liu D, et al. Sex difference in cardiometabolic risk profile and adiponectin expression in subjects with visceral fat obesity. Transl Res. 2010;155:71-7.

26. Gao Z, Zhang X, Zuberi A, Hwang D, Quon MJ, Lefevre M, et al. Inhibition of insulin sensitivity by free fatty acids requires activation of multiple serine kinases in 3 T3-L1 adipocytes. Mol Endocrinol. 2004;18:2024-34.

27. Armoni M, Harel C, Bar-Yoseph F, Milo S, Karnieli E. Free fatty acids repress the GLUT4 gene expression in cardiac muscle via novel response elements. J Biol Chem. 2005;280:34786-95.

28. Holland WL, Miller RA, Wang ZV, Sun K, Barth BM, Bui HH, et al. Receptormediated activation of ceramidase activity initiates the pleiotropic actions of adiponectin. Nat Med. 2011;17:55-63.

29. Plaisance EP, Grandjean PW, Judd RL, Jones KW, Taylor JK. The influence of sex, body composition, and nonesterified fatty acids on serum adipokine concentrations. Metabolism. 2009:58:1557-63.

30. Kishida K, Funahashi T, Shimomura I. Adiponectin as a routine clinical biomarker. Best Pract Res Clin Endocrinol Metab. 2014;28:119-30.

31. Bonora E, Targher G, Alberiche M, Bonadonna RC, Saggiani F, Zenere MB, et al. Homeostasis model assessment closely mirrors the glucose clamp technique in the assessment of insulin sensitivity: studies in subjects with various degrees of glucose tolerance and insulin sensitivity. Diabetes Care. 2000;23:57-63.

32. Bonora E, Kiechl S, Willeit J, Oberhollenzer F, Egger G, Targher G, et al. Prevalence of insulin resistance in metabolic disorders: the Bruneck Study. Diabetes. 1998:47:1643-9.

33. Balkau B, Charles MA. Comment on the provisional report from the WHO consultation. European Group for the Study of Insulin Resistance (EGIR). Diabet Med. 1999;16:442-3. 
34. Sulistyoningrum DC, Gasevic D, Lear SA, Ho J, Mente A, Devlin AM. Total and high molecular weight adiponectin and ethnic-specific differences in adiposity and insulin resistance: a cross-sectional study. Cardiovasc Diabetol. 2013;12:170

35. Hanley AJ, Bowden D, Wagenknecht LE, Balasubramanyam A, Langfeld C Saad MF, et al. Associations of adiponectin with body fat distribution and insulin sensitivity in nondiabetic Hispanics and African-Americans. J Clin Endocrinol Metab. 2007;92:2665-71.

Submit your next manuscript to BioMed Central and take full advantage of:

- Convenient online submission

- Thorough peer review

- No space constraints or color figure charges

- Immediate publication on acceptance

- Inclusion in PubMed, CAS, Scopus and Google Scholar

- Research which is freely available for redistribution 